ARTICLE

Received 17 Dec 2012 | Accepted 18 Apr 2013 | Published 21 May $2013 \quad$ DOl: 10.1038/ncomms2918

\title{
Ice nucleation at the nanoscale probes no man's land of water
}

Tianshu Li', Davide Donadio ${ }^{2} \&$ Giulia Galli ${ }^{3,4}$

At a given thermodynamic condition, nucleation events occur at a frequency that scales with the volume of the system. Therefore at the nanoscale, one may expect to obtain supercooled liquids below the bulk homogeneous nucleation temperature. Here we report direct computational evidence that in supercooled water nano-droplets ice nucleation rates are strongly size dependent and at the nanoscale they are several orders of magnitude smaller than in bulk water. Using a thermodynamic model based on classical nucleation theory, we show that the Laplace pressure is partially responsible for the suppression of ice crystallization. Our simulations show that the nucleation rates found for droplets are similar to those of liquid water subject to a pressure of the order of the Laplace pressure within droplets. Our findings aid the interpretation of molecular beam experiments and support the hypothesis of surface crystallization of ice in microscopic water droplets in clouds.

\footnotetext{
${ }^{1}$ Department of Civil and Environmental Engineering, George Washington University, Washington, District of Columbia 20052, USA. ${ }^{2}$ Max Planck Institute for Polymer Research, Ackermannweg 10, 55128 Mainz, Germany. ${ }^{3}$ Department of Chemistry, University of California, Davis, Davis, California 95616 , USA. ${ }^{4}$ Department of Physics, University of California, Davis, Davis, California 95616, USA. Correspondence and requests for materials should be addressed to T.L. (email: tsli@gwu.edu).
} 
T he physical properties of clouds strongly depend on water nucleation mechanisms and rates, and on the distribution of shapes and sizes of ice particles ${ }^{1,2}$. In particular, the nucleation of small ice particles from the liquid may affect the modulation of solar radiation and hydrological fluxes in the atmosphere ${ }^{3,4}$. Crystallization in nanoscopic ice particles containing from few hundred to tens of thousands of water molecules was probed in the laboratory ${ }^{5-9}$, and the onset of ice crystallization was observed in water clusters containing as few as 275 water molecules ${ }^{6}$. Nucleation and growth of ice within water nano-droplets is also important in exploring water behaviour in so called 'no man's land' ${ }^{\text {to }}$. Rapid crystallization of ice can hardly be avoided in bulk water in this temperature range owing to extremely high crystallization rates. However, reducing sample size leads to less frequent nucleation events, and at the nanoscale one can obtain deeply supercooled water. Indeed, by suspending water clusters containing a few thousand molecules in a supersonic beam, liquid water was supercooled to a temperature as low as $200 \mathrm{~K}^{7}$. This technique also allowed experimentalists to extend the temperature range over which nucleation rates were measured ${ }^{7,8}$.

When interpreting supersonic molecular beam experiments, the nucleation rate of ice is often assumed to be size independent. Although such assumption is valid in bulk and micron size samples, its validity remains to be justified in the nano metre domain. For example, experiments showed that confinement at the nanoscale may modify the bulk phase diagram and shift the coexistence curves ${ }^{11,12}$.

Here, we carry out large-scale molecular simulations to investigate the size dependence of ice nucleation rates within water nano-droplets. Based on the results of our calculations, we propose a simple thermodynamic model that describes the effect of droplet size on ice nucleation rates, and take into account finite size effects.

\section{Results}

Ice nucleation rate. Our molecular dynamics (MD) simulations were carried out using a coarse-grained water model $(\mathrm{mW})^{13}$, which is both computationally efficient and accurate in describing many of the thermodynamic properties of water and ice. The ice nucleation rates were computed by employing the forward flux sampling method ${ }^{14-16}$, which allowed us to collect a large number of nucleation trajectories $(\sim 200)$ at several conditions. We computed ice nucleation rates of droplets, with radii between 2.4 and $6.1 \mathrm{~nm}$, over a wide temperature range from 205 to $240 \mathrm{~K}$, well into 'no man's land'.

Figure 1 shows the calculated ice nucleation rates in the $\mathrm{mW}$ water droplets as a function of their radii at $230 \mathrm{~K}$. We found a strong size dependence of the rates at this temperature, when reducing the radius from 4.9 to $2.4 \mathrm{~nm}$ : the computed nucleation rate decreased by eight orders of magnitude, from $1.08 \pm 0.84 \times 10^{14}$ to $1.10 \pm 0.99 \times 10^{6} \mathrm{~m}^{-3} \mathrm{~s}^{-1}$. On the other hand, as the radius of the droplet becomes larger than $\sim 5 \mathrm{~nm}$, the computed nucleation rate becomes virtually indistinguishable from that of the bulk liquid at the same temperature ${ }^{17}$, which suggests size dependence diminishes beyond this size.

To understand whether the observed size dependence of ice nucleation rates persists at different temperatures, we carried out extensive simulations over a wide temperature range, from 205 to $240 \mathrm{~K}$. Figure 2 displays the calculated nucleation rate as a function of temperature for bulk water and droplets with radius of 3.1 and $2.4 \mathrm{~nm}$, respectively. At low temperature $(T<210 \mathrm{~K})$, the calculated ice nucleation rates differ only by one order of magnitude for different sizes, suggesting the suppression of the size dependence in ice nucleation rates in this regime. Such

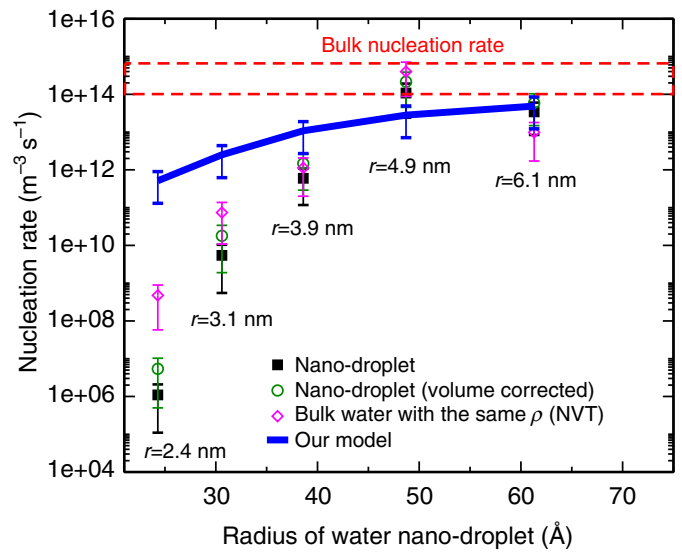

Figure 1 | Size dependence of ice nucleation rates in the $\mathbf{m W}$ water droplets at $230 \mathrm{~K}$. The solid black squares denote the calculated ice nucleation rate within the $\mathrm{mW}$ droplets, the dash lines indicate the computed ice nucleation rate in the $\mathrm{mW}$ bulk water, the green circles denote the corrected ice nucleation rate by using the effective volume, and the purple diamonds represent the calculated nucleation rate in bulk liquid with the density matching that of the corresponding droplet. The effective volume of the droplet is defined as the total volume minus the surface-like volume (see Supplementary Note 3). The thick blue line represents the predicted nucleation rate based on our model (equation (3)). The statistical uncertainty of the computed nucleation rate is mainly due to the error in the calculated growth probability $P\left(\lambda_{n} \mid \lambda_{0}\right)$ (see Methods) that is attributed to both the variance of the binomial distribution of $N$, i.e., the number of configurations collected at each interface, and the landscape variance of each starting configuration at each interface ${ }^{15}$. The error bar in the predicted rate from our model simply reflects the error bar in the calculated bulk nucleation rate.

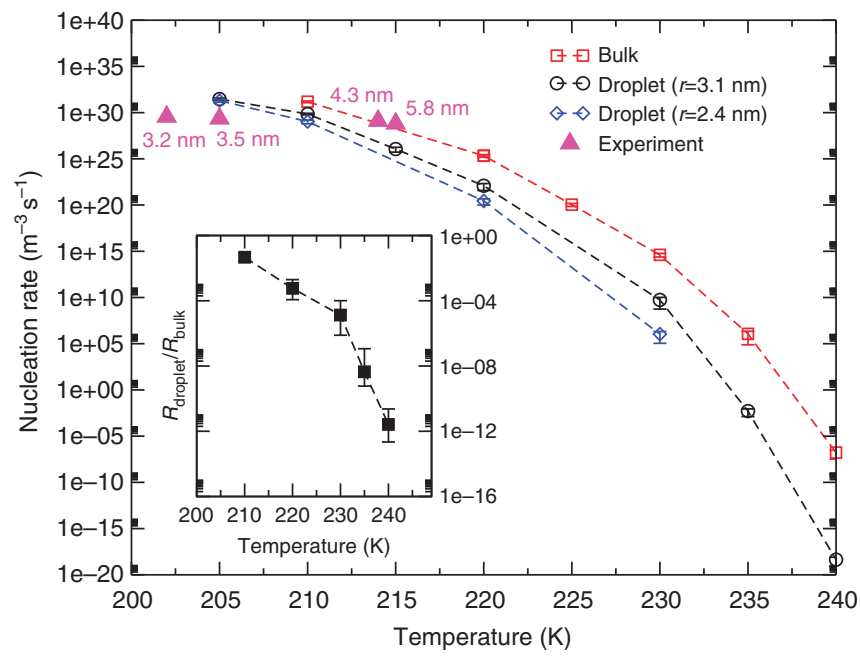

Figure 2 | Temperature dependence of ice nucleation rates in the $\mathbf{m W}$ water model. The calculated nucleation rate in bulk water, droplet with radius of $3.1 \mathrm{~nm}$, and droplet with radius of $2.4 \mathrm{~nm}$ are represented by red squares, black circles and blue diamonds, respectively. Experimental data (represented by triangles) are from Manka et al. ${ }^{8}$ Inset shows the temperature variation of the ratio of the computed ice nucleation rate between water droplet with radius of $3.1 \mathrm{~nm}$ and bulk water.

insensitivity to size is in quantitative agreement with recent experimental measurements of nucleation rates in water nanodroplets with radii between 3.2 and $5.8 \mathrm{~nm}$, and between 202 and $215 \mathrm{~K}^{8}$. 
At higher temperature, our simulations clearly indicate a strong size dependence of ice nucleation rates, as shown, for example, by the ratio between the ice nucleation rate in a 3.1-nm droplet and in bulk, $R_{\text {droplet }} / R_{\text {bulk, }}$, plotted as a function of temperature. As shown in the inset of Fig. 2, a sharp decrease of $R_{\text {droplet }} / R_{\text {bulk }}$ is observed as the temperature is increased. In particular, the decrease of $R_{\text {droplet }} / R_{\text {bulk }}$ yields the suppression of ice nucleation rates by over 12 orders of magnitude in $3.2 \mathrm{~nm}$ droplets, with respect to bulk water at $240 \mathrm{~K}$.

Both theory ${ }^{18}$ and experiments ${ }^{19,20}$ showed that the melting temperature of ice is decreased by confinement. In particular, recent simulations showed that the equilibrium melting temperature of ice nanoparticles follows the Gibbs-Thomson equation $^{21,22}$. Although the decrease of nucleation rates with size may appear to correlate with the decrease in melting temperature with size, our results cannot be explained in terms of a shift of phase equilibrium due to confinement (see Supplementary Note 1 and Supplementary Fig. S1). For example, in the $4.9 \mathrm{~nm}$-droplet, the calculated ice nucleation rate at $230 \mathrm{~K}$ is already virtually indistinguishable from that of the bulk liquid, whereas its melting temperature was determined to be $255 \pm 2 \mathrm{~K}^{22}$, that is, about $20 \mathrm{~K}$ lower than the melting temperature of bulk ice at ambient pressure.

Effects of liquid surface on ice nucleation rates. To shed light on the origin of the size dependence of ice nucleation rates in water nano-droplets, we considered the role played by the liquid surface. In particular, we consider its three major effects on the thermodynamics and kinetics of the liquid-solid phase transition: (1) The structural disorder of molecules or atoms at the surface usually disrupts the crystalline order. Therefore, a liquid surface is generally not considered as a preferential site for crystal nucleation. In fact, although melting initiated at the surface has been often observed in experiments, surface freezing is much less common $^{23,24}$. (2) For tetrahedral liquids exhibiting a negative slope of their melting lines $\left(\mathrm{d} T /\left.\mathrm{d} P\right|_{\text {coexist }}<0\right)$, for instance silicon and water, we proposed that the presence of a liquid-vapour surface enhances the crystallization rate: indeed in these systems the density decrease upon crystallization may be better accommodated in the vicinity of the liquid surface ${ }^{16,25}$. (3) In a liquid nano-droplet, due to the curvature of the surface, there exists a non-negligible Laplace pressure, which can be expressed as $p_{\mathrm{L}}=2 \gamma_{\mathrm{lv}} / r$, where $\gamma_{\mathrm{lv}}$ and $r$ are the liquid-vapour surface tension and the radius of the droplet, respectively.

Effect (2) and (3) can be combined by considering the role of a small pressure $p$ on crystal nucleation rates. For effect (2), a negative pressure $p=2 / 3 p_{\mathrm{T}}$ (where $p_{\mathrm{T}}$ is the lateral pressure component parallel to the liquid surface), is expected due to surface tension (see Supplementary Note 2). For effect (3), a positive pressure $p=p_{\mathrm{L}}$ is exerted onto the interior of the liquid droplet. To further quantify this pressure effect, we built a simple model based on classical nucleation theory $(\mathrm{CNT})$ by considering the change in the driving force for nucleation due to a small pressure. In particular, considering a spherical critical nucleus, as identified approximately in our simulation (see Supplementary Note 4 and Supplementary Fig. S7), the nucleation rate in the bulk liquid at zero pressure $\left(R_{\mathrm{b}}\right)$ can be expressed by the following equation:

$$
R_{\mathrm{b}}=A \exp \left[-\frac{16 \pi \gamma_{\mathrm{ls}}^{3}}{3 k_{\mathrm{B}} T\left(\rho \Delta \mu_{\mathrm{b}}\right)^{2}}\right]
$$

where $A$ is a kinetic pre-factor, $\gamma_{\mathrm{ls}}$ is the solid-liquid surface tension, $\rho$ is the density of the liquid, and $\Delta \mu_{\mathrm{b}}$ is the chemical potential difference between the metastable liquid and the solid under zero pressure at the temperature $T$. The nucleation rate $R_{\mathrm{p}}$ in the presence of a small pressure $p$ (that is, $p \ll \Delta \mu_{\mathrm{b}} / \Delta v$, where $\Delta v=v_{1}-v_{\mathrm{s}}$ is the molar volume difference between liquid and solid at pressure $p$ ) may be evaluated; the chemical potential variation is:

$$
\Delta \mu_{\mathrm{p}}=\Delta \mu_{\mathrm{b}}+p \Delta v
$$

It can be shown (see Supplementary Note 2) that the ratio $R_{\mathrm{p}} / R_{\mathrm{b}}$ is given by:

$$
\frac{R_{\mathrm{p}}}{R_{\mathrm{b}}}=\exp \left[\frac{32 \pi}{3 k_{\mathrm{B}} T \rho^{2}}\left(\frac{\gamma_{\mathrm{ls}}}{\Delta \mu_{\mathrm{b}}}\right)^{3} p \Delta v\right]
$$

If the pressure $p$ and the molar volume difference $\Delta v$ are of the same sign, the term inside the exponential of equation (3) is positive, thus the presence of pressure leads to an enhancement of the nucleation rate. If their signs are opposite, then the nucleation rate is suppressed. This CNT-based model also suggests that the quantitative change in the nucleation rate depends not only on the magnitude of $p \Delta v$, but also on the ratio of $\gamma_{\mathrm{ls}} / \Delta \mu_{\mathrm{b}}$, and on the temperature $T$. According to our model, in tetrahedral liquids with a negative sign of their melting line in the $P, T$ phase diagram, that is, $\Delta v<0$, the negative pressure $(p<0)$ in the vicinity of the liquid surface, arising from surface tension, is expected to enhance crystal nucleation rates.

Before applying our model to water droplets, we validated it for the case of $\mathrm{Si}$ and water at the macroscopic scale. Using the molecular volumes and the surface tension of the liquid-vacuum interface $^{16}$ for silicon modelled with the Tersoff potential ${ }^{26}$, we obtained an enhancement of the nucleation rate in the subsurface liquid layer with respect to that of the bulk by a factor of $\sim 10^{2}$ at the temperature of $0.95 T_{\mathrm{m}}$ (see Supplementary Note 2). This estimate is in agreement with previous numerical results that showed a thousand fold increase in the calculated nucleation rate in the silicon liquid slabs ${ }^{16}$. On the other hand, from the thermodynamic quantities of the $\mathrm{mW}$ water model ${ }^{13,17}$, we find that our CNT model predicts only a marginal increase of ice nucleation rate in the vicinity of flat water surfaces, for example, $R_{\mathrm{p}} / R_{\mathrm{b}} \sim 3$ at $230 \mathrm{~K}$. To confirm the prediction of the $\mathrm{mW}$ water model, we computed ice nucleation rates in supercooled water slabs using 4,096 $\mathrm{mW}$ water molecules, and indeed found no significant difference in the computed nucleation rates from those in bulk water (see Supplementary Fig. S5).

Now we turn our attention to the $\mathrm{mW}$ water nano-droplets at $230 \mathrm{~K}$. The curvature of the liquid/vacuum interface induces the Laplace pressure $p_{\mathrm{L}}$. To estimate the magnitude of $p_{\mathrm{L}}$, we computed the liquid-vacuum surface tension $\gamma_{\mathrm{lv}}=69.2 \mathrm{~mJ} \mathrm{~m}^{-2}$ for the $\mathrm{mW}$ model at $230 \mathrm{~K}$ by employing the Kirkwood and Buff $\operatorname{method}^{27}$. Neglecting the curvature dependence of $\gamma_{\mathrm{lv}}$, we obtained a variation of $p_{\mathrm{L}}$ with respect to the radius of the droplets, as shown in Fig. 3a. On the other hand, the internal pressure of the $\mathrm{mW}$ droplets can also be estimated from isothermal ensemble simulations of $\mathrm{mW}$ bulk water at a density matching the one of the droplets. The two methods yield consistent results: Fig. 3a shows that the internal pressure of water nano-droplets follows closely the directly computed Laplace pressure $p_{\mathrm{L}}$. The induced internal pressure within the $\mathrm{mW}$ water nano-droplets is of the order of several hundred bars.

A positive pressure $p$ increases the density of water (see Fig. 3b). Therefore, as the compressibility of ice is negligibly small compared to that of water, the density difference between the liquid and the solid increases as the droplet becomes smaller (see Supplementary Fig. S7). Thus the nucleation rate of ice in droplets is expected to decrease with the radius due to the increasing magnitude of the $p \Delta v$ term. To verify this analysis 

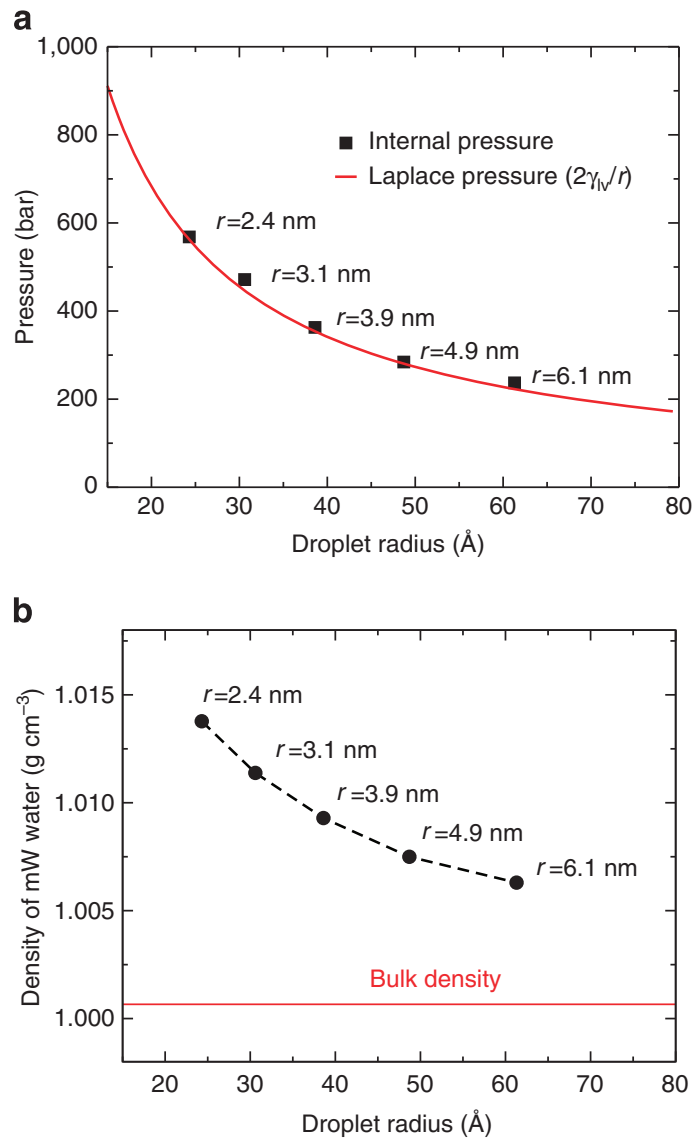

Figure 3 | Pressure and density of water within the $\mathbf{m W}$ nano-droplet

at $230 \mathrm{~K}$. (a) Variation of internal pressure with the radius of water nano-droplet. The Laplace pressure $p_{\mathrm{L}}=2 \gamma_{\mathrm{IV}} / r$ estimated based on $\gamma_{\mathrm{IV}}$ is plotted by the red solid line, while the pressure obtained from simulation is represented by black squares. (b) Variation of liquid density with the radius of droplet. The red line represents the density of water at ambient pressure.

numerically, we conducted additional simulations in dense $\mathrm{mW}$ bulk water, that is, with density matching that of the corresponding $\mathrm{mW}$ water nano-droplet at $230 \mathrm{~K}$. Figure 1 shows the overall agreement on the computed ice nucleation rates between the dense bulk liquid and the water nano-droplets. An exception is the smallest droplet $(r=2.4 \mathrm{~nm})$, where the size of the critical nucleus at this temperature was determined to be around 570 water molecules (see Supplementary Fig. S6) by the committor probability analysis ${ }^{28}$. Therefore, a significant portion of the critical nucleus overlaps with the surface region (see Supplementary Fig. S8), where the disordered arrangement of molecules further suppresses the formation of ice. We thus conclude that ice nucleation rates within the $\mathrm{mW}$ water nanodroplets can be reproduced well by simulations in bulk $\mathrm{mW}$ water under pressure, tuning the density so that it corresponds to that of the droplets.

The simulation results can also be well reproduced by our model, for large droplets $(r>3.9 \mathrm{~nm})$. By using bulk nucleation rate, we calculate the variation of ice nucleation rate with the radius of the droplet based on equation (3) at $230 \mathrm{~K}$, as shown in Fig. 1. The good agreement between our model and the simulation results is achieved at large sizes of the water droplet where the internal pressure is small. As the size of the droplet decreases (and thus the internal pressure becomes greater), the calculated nucleation rates become increasingly lower than those predicted by our model. The deviation can be attributed to the

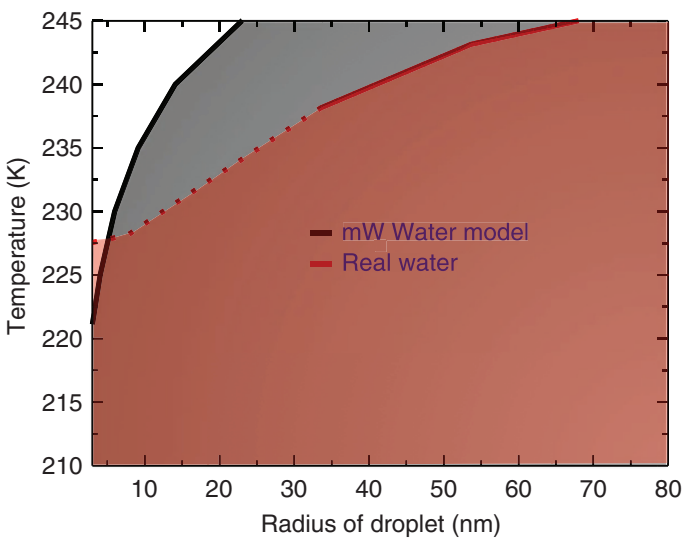

Figure 4 | Temperature-radius diagram of ice nucleation rates in supercooled water nano-droplets. The boundary between the bulk-like (shaded area) and droplet-like (unshaded area) region is constructed by solving for the radius $r$ in the equation $R_{\mathrm{p}} / R_{\mathrm{b}}=1 / 5$, that is, by satisfying the condition that the ice nucleation rate in water droplets is one fifth of that of bulk water, at each temperature (see equation (3)). Black and red colours represent the $\mathrm{mW}$ water model and real water, respectively. The density of bulk water below $238 \mathrm{~K}$ was not measured directly in experiment ${ }^{29}$, but was extrapolated down to $227 \mathrm{~K}^{30}$. The boundary for real water at this temperature range is thus represented by a dotted line.

large $p \Delta v$ term that can no longer be treated as a small perturbation in equation (3). In addition, the variation of $\gamma_{\mathrm{ls}}$ and $\Delta \mu_{\mathrm{b}}$ with pressure needs to be included in the model as the pressure becomes large.

Finally, the temperature dependence of the ratio between ice nucleation rates in bulk and droplets can be naturally accounted for by our model. At low temperature, the driving force for ice nucleation $\Delta \mu_{\mathrm{b}}$ becomes much larger than $p \Delta v$, thus the curvature induced Laplace pressure only has a negligible role in ice nucleation rates. In fact, our model predicts that the $\mathrm{mW}$ bulk ice nucleation rate is only about three times larger than that in the $\mathrm{mW}$ droplet of $3.1 \mathrm{~nm}$ in radius at $210 \mathrm{~K}$, consistent with our simulation results (inset of Fig. 2). As a summary of the results of our thermodynamic model, we constructed a temperature-radius diagram (Fig. 4) using the thermodynamic data of the $\mathrm{mW}$ water model $^{13}$ and real water ${ }^{29,30}$, to illustrate both the temperature and size effects on ice nucleation rate in supercooled water nanodroplets. At a given temperature, the diagram suggests that water droplets with radius greater than that indicated by the boundary would exhibit a bulk-like behaviour, that is, ice nucleation rates in droplets are within the statistical uncertainty of the calculated bulk ice nucleation rate.

\section{Discussion}

Our molecular simulations demonstrate that ice nucleation rates of the $\mathrm{mW}$ water nano-droplets are both size and temperature dependent. In general, we find that the Laplace pressure induced by the curved liquid-vapour interface suppresses the crystallization of ice, yielding orders of magnitude reduction in nucleation rates relative to that in bulk water. A simple model describing the effect of the $p \Delta v$ term on nucleation rates offers a reasonable explanation for the reduction of the nucleation rate in water nano-droplets: a positive $p \Delta v$ promotes crystallization, whereas a negative $p \Delta v$ suppresses crystallization. The quantitative change in the nucleation rate depends on other thermodynamic quantities in equation (3). In smaller droplets, as the critical size of ice nuclei increases (Supplementary Fig. S6), one expects a critical droplet size, comparable to that of critical ice 
nuclei, below which ice crystallization can be completely suppressed. Indeed recent experiments ${ }^{6}$ identified the onset of ice crystallization to occur in a water cluster containing 275 water molecules at $90 \sim 115 \mathrm{~K}$, and recent simulation ${ }^{22}$ based on the $\mathrm{mW}$ water model have shown that ice formation can be avoided in a droplet of $1 \mathrm{~nm}$ in radius at $150 \mathrm{~K}$. We note that our numerical results were obtained based on the $\mathrm{mW}$ water model that underestimates the density difference $(2.3 \%)$ between ice and water at $T_{\mathrm{m}}$ (as compared with the experimental value of $8.2 \%{ }^{29}$. See Supplementary Fig. S4). Therefore, the quantitative effect of the $p \Delta v$ term on ice nucleation rate should be more prominent in real water. This would lead to two consequences: first, in real water nano-droplet, the suppression of ice nucleation rates is expected to be more pronounced than found here with the $\mathrm{mW}$ model, particularly at modest supercooling. Second, in real micrometre water droplets, where the effect of Laplace pressure can be neglected, ice nucleation rate may be also significantly affected by the presence of a water/vapour interface. For example, our model predicts an increase of the ice nucleation rate by about three orders of magnitude at $240 \mathrm{~K}$ in real micrometre water droplet due to the presence of a water/vapour surface. It thus supports the hypothesis of surface crystallization of water droplets suspended in clouds ${ }^{31}$.

\section{Methods}

MD simulations. MD simulations were performed based on the $\mathrm{mW}$ water model with a time step of $2 \mathrm{fs}$. Simulations in droplets were conducted within an isothermal canonical ensemble (NVT) with a Nose-Hoover thermostat with relaxation time of 1 ps. As periodic boundary conditions were used in all the simulations, the liquid droplet was obtained by adding about $40 \AA$ to each of the dimensions of the cubic unit cell of the well-equilibrated bulk water, and annealing to the target temperature for over $10 \mathrm{~ns}$. The droplets with the radii of 2.4, 3.1, 3.9, 4.9 and $6.1 \mathrm{~nm}$ contain $2,048,4,096,8,192,16,386$ and 32,768 water molecules, respectively. The radius of the droplet is determined from the calculated density profile (Supplementary Fig. S2) by locating the position corresponding to $1 / 2$ of the bulk density. The water slab was obtained by adding about $20 \AA$ along $z$ to the equilibrium configuration of the bulk water cell containing 4,096 molecules.

Calculation of ice nucleation rate. The nucleation rate was computed by applying the forward flux sampling method ${ }^{14}$. In this approach, the rate constant is given by $R=\dot{\Phi}_{\lambda_{0}} \prod_{i=1}^{n} P\left(\lambda_{i} \mid \lambda_{i-1}\right)$, where $\dot{\Phi}_{\lambda_{0}}$ and $P\left(\lambda_{i} \mid \lambda_{i-1}\right)$ are the flux rate crossing the first interface $\lambda_{0}$ and the probability for a trajectory starting from the $(i-1)^{\text {th }}$ interface and successfully reaching the $i^{\text {th }}$ interface, respectively. The interface is defined based on the order parameter $\lambda$ that is the number of the water molecules contained in the largest ice cluster. The detail of this approach can be found in Li et al. ${ }^{32}$ A local order parameter $q_{6}$ is employed to differentiate ice-like molecule from water-like molecule: a water molecule with $q_{6}>0.5$ is defined as ice-like. The definition and the robustness of $q_{6}$ are described in Li et al. ${ }^{17}$ To compute the flux rate $\dot{\Phi}_{\lambda_{0}}$, we carried out direct MD simulation $(t \sim 100 \mathrm{~ns})$ and counted the number $(N=500)$ of direct crossings of the first interface $\lambda_{0}$ from the liquid configuration with $\lambda<\lambda_{\mathrm{A}}$. The flux rate $\dot{\Phi}_{\lambda_{0}}$ is obtained by $N /(t V)$, where $V$ is the volume of the liquid. To compute the growth probability $P\left(\lambda_{i} \mid \lambda_{i-1}\right)$, a large number of MD trial runs $M_{i-1}$ were fired from the interface $i-1$, until $N(=110-220)$ successful crossings to the next interface $i$ were collected, and $P\left(\lambda_{i} \mid \lambda_{i-1}\right)$ is given by $N / M_{i-1}$. As the size of the ice nucleus grows large enough, that is, well beyond the critical size $\lambda_{c}$, the growth probability $P\left(\lambda_{i} \mid \lambda_{i-1}\right)$ reaches one, yielding the converged nucleation rate $R$. The calculated growth probability $P\left(\lambda_{n} \mid \lambda_{0}\right)$ as a function of nucleus size $\lambda$ for the $\mathrm{mW}$ water droplets with various radii at $230 \mathrm{~K}$ is shown in Supplementary Fig. S9. The statistical uncertainty of the computed nucleation rate is mainly due to the error in the calculated growth probability $P\left(\lambda_{n} \mid \lambda_{0}\right)$ that is attributed to both the variance of the binomial distribution of $N$, i.e., the number of configurations collected at each interface, and the landscape variance of each starting configuration at each interface ${ }^{15}$. The typical error bar of the computed nucleation rate is within $75-90 \%$ of the calculated rate. The total simulation length of each nucleation rate is of the order of a microsecond.

\section{References}

1. Baker, M. Cloud microphysics and climate. Science 276, 1072-1078 (1997).

2. Cantrell, W. \& Heymsfield, A. Production of ice in tropospheric clouds: a review. B Am. Meteorol. Soc. 86, 795-807 (2005).

3. Zender, C. S. \& Kiehl, J. T. Radiative sensitivities of tropical anvils to small ice crystals. J. Geophys. Res. Atmos. 99, 25869-25880 (1994)

4. Hegg, D. A. \& Baker, M. B. Nucleation in the atmosphere. Rep. Prog. Phys. 72, 056801 (2009)
5. Buch, V., Bauerecker, S., Devlin, J. P., Buck, U. \& Kazimirski, J. Solid water clusters in the size range of tens-thousands of H2O: a combined computational/ spectroscopic outlook. Int. Rev. Phys. Chem. 23, 375-433 (2004).

6. Pradzynski, C. C., Forck, R. M., Zeuch, T., Slavicek, P. \& Buck, U. A fully sizeresolved perspective on the crystallization of water clusters. Science 337, 1529-1532 (2012).

7. Huang, J. \& Bartell, L. Kinetics of homogeneous nucleation in the freezing of large water clusters. J. Phys. Chem. 99, 3924-3931 (1995).

8. Manka, A., Pathak, H., Tanimura, T., Wölk, J., Strey, R. \& Wyslouzil, B. Freezing water in no-man's land. Phys. Chem. Chem. Phys. 14, 4505-4516 (2012).

9. Delzeit, L. \& Blake, D. A characterization of crystalline ice nanoclusters using transmission electron microscopy. J. Geophys. Res. 106, 33371-33379 (2007).

10. Debenedetti, P. Supercooled and glassy water. J. Phys. Condens. Mat. 15, R1669-R1726 (2003)

11. Goldstein, A. N., Echer, C. M. \& Alivisatos, A. P. Melting in semiconductor nanocrystals. Science 256, 1425-1427 (1992).

12. Jacobs, K., Zaziski, D., Scher, E. C., Herhold, A. B. \& Alivisatos, A. P. Activation volumes for solid-solid transformations in nanocrystals. Science 293, 1803-1806 (2001).

13. Molinero, V. \& Moore, E. B. Water modeled as an intermediate element between carbon and silicon. J. Phys. Chem. B 113, 4008-4016 (2009).

14. Allen, R. J., Frenkel, D. \& Wolde, P. R. T. Simulating rare events in equilibrium or nonequilibrium stochastic systems. J. Chem. Phys. 124, 024102 (2006).

15. Allen, R. J., Frenkel, D. \& Wolde, P. R. T. Forward flux sampling-type schemes for simulating rare events: efficiency analysis. J. Chem. Phys. 124, 194111 (2006).

16. Li, T., Donadio, D., Ghiringhelli, L. M. \& Galli, G. Surface-induced crystallization in supercooled tetrahedral liquids. Nat. Mater. 8, 726-730 (2009).

17. Li, T., Donadio, D., Russo, G. \& Galli, G. Homogeneous ice nucleation from supercooled water. Phys. Chem. Chem. Phys. 13, 19807-19813 (2011).

18. Limmer, D. T. \& Chandler, D. Phase diagram of supercooled water confined to hydrophilic nanopores. J. Chem. Phys. 137, 044509 (2012).

19. Schreiber, A., Ketelsen, I. \& Findenegg, G. H. Melting and freezing of water in ordered mesoporous silica materials. Phys. Chem. Chem. Phys. 3, 1185-1195 (2001).

20. Jähnert, S., Chávez, F. V. \& Schaumann, G. E. Melting and freezing of water in cylindrical silica nanopores. Phys. Chem. Chem. Phys. 10, 6039-6051 (2008).

21. Pan, D., Liu, L.-M., Slater, B., Michaelides, A. \& Wang, E. Melting the ice: on the relation between melting temperature and size for nanoscale ice crystals. ACS Nano 5, 4562-4569 (2011).

22. Johnston, J. C. \& Molinero, V. Crystallization, melting, and structure of water nanoparticles at atmospherically relevant temperatures. J. Am. Chem. Soc. 134, 6650-6659 (2012).

23. van der Veen, J. Melting and freezing at surfaces. Surf. Sci. 433, 1-11 (1999).

24. Pluis, B., Frenkel, D. \& Vanderveen, J. Surface-induced melting and freezing ii. a semiempirical landau-type model. Surf. Sci. 239, 282-300 (1990).

25. Vrbka, L. \& Jungwirth, P. Homogeneous freezing of water starts in the subsurface. J. Phys. Chem. B. 110, 18126-18129 (2006).

26. Tersoff, J. Modeling solid-state chemistry: interatomic potentials for multicomponent systems. Phys. Rev. B 39, 5566-5568 (1989).

27. Kirkwood, J. G. \& Buff, F. P. The statistical mechanical theory of surface tension. J. Chem. Phys. 17, 338-343 (1948).

28. Bolhuis, P. G., Chandler, D., Dellago, C. \& Geissler, P. L. Transition path sampling: throwing ropes over rough mountain passes, in the dark. Annu. Rev. Phys. Chem 53, 291-318 (2002).

29. Hare, D. E. \& Sorensen, C. M. The density of supercooled water. II. Bulk samples cooled to the homogeneous nucleation limit. J. Chem. Phys. 87, 4840-4845 (1987).

30. Speedy, R. J. Thermodynamic properties of supercooled water at $1 \mathrm{~atm}$. J. Phys. Chem. 91, 3354-3358 (1987).

31. Tabazadeh, A., Djikaev, Y. \& Reiss, H. Surface crystallization of supercooled water in clouds. Proc. Natl Acad. Sci. USA 99, 15873-15878 (2002).

32. Li, T., Donadio, D. \& Galli, G. Nucleation of tetrahedral solids: a molecular dynamics study of supercooled liquid silicon. J. Chem. Phys. 131, 224519 (2009).

\section{Acknowledgements}

We gratefully thank Pablo Debenedetti, Austen Angell, Valeria Molinero, Dennis Klug and Thomas Koop for fruitful discussions. T.L. acknowledges support from the Sloan Foundation through the Deep Carbon Observatory and The American Chemical Society Petroleum Research Fund. T.L. thanks the Department of Civil and Environmental Engineering at the George Washington University for computing support. G.G. acknowledges support from DOE-CMSN, under Grant number DE-SC0005180. 


\section{Author contributions}

T.L., D.D., and G.G. designed the research project. T.L. performed and analysed simulations. T.L., D.D. and G.G. wrote the paper.

\section{Additional information}

Supplementary Information accompanies this paper at http://www.nature.com/ naturecommunications
Competing financial interests: The authors declare no competing financial interests.

Reprints and permission information is available online at http://npg.nature.com/ reprintsandpermissions/

How to cite this article: $\mathrm{Li}$, T. et al. Ice nucleation at the nanoscale probes no man's land. Nat. Commun. 4:1887 doi: 10.1038/ncomms2918 (2013). 